\title{
Mechanical cutting Technique of the Steel Tower Segments of Long-span Cable-Supported Bridge
}

\author{
Wang Hui ${ }^{a}$, Zhang Qinglei, He Shiqin and Yan Fei \\ College of Civil Engineering, North China University of Technology, China \\ awanghuitju@126.com
}

\begin{abstract}
Keywords: Cable-Supported Bridge, Steel tower, Mechanical cutting Technique, Computer Aided Manufacturing

Abstract. When the steel structure is used as the main tower of long-span cable-supported bridge, one big difficult that we must face is the precision control of the steel tower segment. In order to meet fabrication and erecting precision requirements, high precision of the mechanical cutting of each segment end face is required. Whereas there are numerous factors affecting processing precise, if one of them is ignored, the precision requirement cannot be satisfied. This paper introduced the development of the mechanical cutting technique and application of the computer aided mechanical cutting technique in modern bridge engineering.
\end{abstract}

\section{Introduction}

The modern suspension bridge and cable stayed bridge have been developed for more than100 years and 50years respectively. From the Williamsburg Bridge to the Akashi-Kaikyo Bridge and from the StrÖmsund Bridge to the Tataro Bridge, most of them adopted steel tower. In China, scores of long-span suspension bridges and cable stayed bridges have been built since 1990s and most of them adopted the concrete tower because of the lower cost of the tower itself and the tradition of the designer and builder. However, in this century, with the increase in China's steel production, the steel tower becomes another choice in China because of its advantages such as light deadweight, quick construction and strong resistance in earthquake [1]. Main tower is the important component of the suspension bridge and cable stayed bridge. Generally, long-span Cable-Supported Bridge main tower can exceed 200 meters.

When the steel structure is used as the main tower, it must be divided into many segments to fabricate, and during erecting the segments are connected by the metal contact in end face and high tensile bolts connection [2]. Because the precision of the mechanical cutting affects the shape of the whole tower directly, thereby affects the paths of the forces through the structure, high precision of the mechanical cutting of each segment end face is required. Along with the progress of social technology conditions, the mechanical cutting technique of the steel tower segment has been greatly developed, now computer aided mechanical cutting technique has provided strong support for the realization of high precision requirements of the modern steel tower. In this paper, the development of the steel tower segment mechanical cutting technique has been reviewed and the computer technique used in this filed is introduced.

\section{Influencing factors of the mechanical cutting precision of the modern steel tower}

The important function of the cable-supported bridge main towers related to the geometry of the main tower is to transfer the force from the stay cable or main cable to the foundations. Because of the big size, the steel tower has to be divided into many segments in factory and erected segment by segment at bridge site [2-3]. The shape of the steel tower is difficult to adjust and the fabrication error of each segment will be accumulated and propagated during erection. At the same time, In order to ensure reliable power transmission between adjacent segments, a high end face metal contact rate must be obtained. Accordingly, high fabrication precision of each segment must be achieved. For example, table 1 presents precision standard of Nanjing third Yangtze River Bridge steel tower [4], whereas 
there are numerous influencing factors of the mechanical cutting, if one of them is ignored, the precision requirement cannot be satisfied. So it is necessary to analysis the factors that influence the mechanical cutting precision, and the corresponding countermeasure should be taken. The main factors that influence the cutting precision are as follows:

1. The design of the tower, the properties of the steel and the tolerance of the thickness of steel plates.

2. The precise of machine tool and the cutting workmanship.

3. The method to get the axis of the segment and the cutting style.

4. The influence the temperature difference and the vibration of surroundings.

5. The influence of the geometric shape of the steel tower segment.

6. The procedure error caused by measurement error and the distortion caused by deadweight of the steel tower segment.

Table 1 Precision standard of Nanjing third Yangtze River bridge steel tower

\begin{tabular}{c|c|c}
\hline & Item & Tolerance error \\
\hline \multirow{4}{*}{ Single segment } & Length of the segment/mm & \pm 2.0 \\
\cline { 2 - 3 } & Torsion/mm & \pm 3.0 \\
\cline { 2 - 3 } & $\begin{array}{c}\text { Inclination error between the } \\
\text { axes and end face }\end{array}$ & $20^{\prime \prime}$ \\
\cline { 2 - 3 } & Plainness of end face/mm & 0.25 \\
\hline \multirow{3}{*}{ Preassembly } & Total Length/mm & $\pm 2.0 \times \mathrm{n}(\mathrm{n}$-Number of the segment $)$ \\
\cline { 2 - 3 } & Vertical degree & $1 / 10000$ \\
\cline { 2 - 3 } & Slab staggering/mm & 2 \\
\hline
\end{tabular}

\section{The Development of Mechanical cutting Technique of steel tower segment [5-8]}

During $1970 \sim 1980$ s, the weld structure was widely used in the long-span bridge main tower such as Kanmonkyo Bridge and Seto-ohashi Bridge. In these bridges, after the segment was welded, plumbs were put on the 4 sides of the segment and the distance between the middle points of the lower end face sides and the vertical lines were minimized by adjusting the jack support, at the same time the vertical lines were taken as the axes of the fabricated segments. Then, the segments were positioned horizontally to be cut by the machine tool. The machined segments were positioned vertically and measured. At last the segments were positioned horizontally again and the segments preassembly was done. All the mechanical cutting was executed in the workshop to avoid the effect of the variations of the temperature.

Compared with the early built bridges, the steel tower of Akashi kaikyo Bridge built in 1998 has a larger scale weighing about 400t every segment. It is very difficult to change position and attitude of the segment. Therefore the segments are positioned horizontally during the whole manufacturing process. The following control systems were adopted and processed by computer precision management system.

1. The laser theodolite was used to measure the 3D shape of the segment and the result was input into the computer.

2. Using the principle of least squares method, segmental longitudinal axis and the cutting plane which is perpendicular to longitudinal axis were obtained.

3. Computer controlled hydraulic support system to eliminate the error caused by the weight of the segment was adopted.

4. The CNC machine tools were used to cut the end face of the segment. 


\section{The application of the Computer Aided Mechanical cutting Technique on Nanjing third Yangzi River Bridge}

Nanjing Yangtze River 3rd bridge was completed in 2005. It is the first bridge that uses the steel structure as the main tower in China. The steel tower is $215 \mathrm{~m}$ high as shown in Fig.1. The segment is shown in Fig.2.

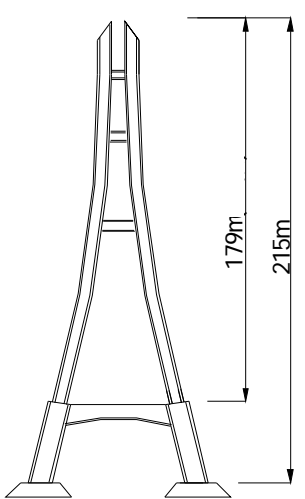

Fig.1 Elevation of Steel Tower of Nanjing 3rd Yangtze River Bridge

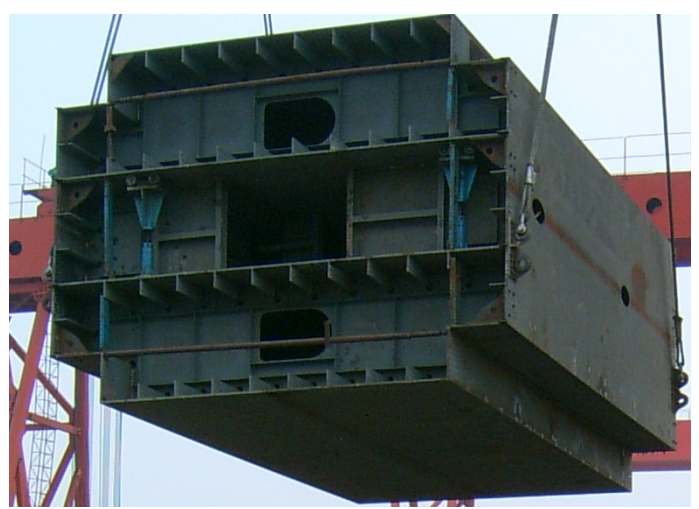

Fig.2 Steel tower segments

After welding, Nanjing Yangtze River 3rd bridge Steel tower segments were positioned horizontally. Then the 3D shape was measured and the longitudinal axis and the cutting plane were calculated by the computer. The machine tool is shown in Fig. 3 and the milling cutter is shown in Fig. 4. After that, the mechanical cutting was executed in the workshop. Due to the limited conditions of the workshop, the section moved to the outdoor to finish the preassembly [8-9].

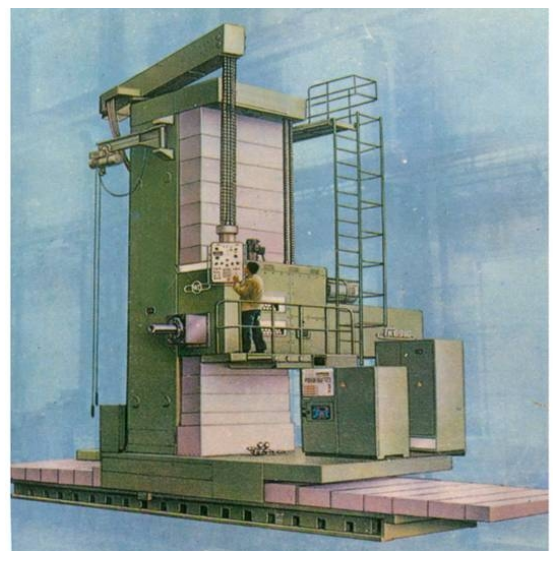

Fig.3 Machine tool for Steel Tower

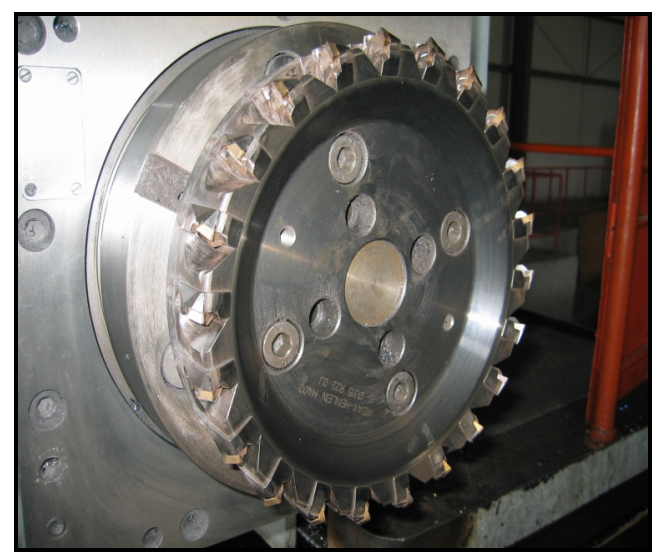

Fig.4 WIDIA milling cutter

Compared with the Akashi Strait Bridge Tower, Nanjing bridge steel tower manufacturing has the following differences.

1. Compared with the Akashi kaikyo Bridge, Nanjing bridge tower segments are smaller and they are overall welding structures, thereby the processing difficulty is reduced.

2. The feature points that can determine the whole shape of the fabricated segment were analyzed and the API High precision measuring instrument was adopted to obtain the 3D coordinate values of the feature points. $6 \mathrm{D}$ least squares principle was used to calculate the axis and the cutting planes of the segment. These provide the conditions for the realization of the 3-D numerical pre assembly.

3. Computer controlled hydraulic support system is used eliminate the error caused by the weight of the segment. The large NC milling machine is used for controlling planeness accuracy. 
4. Due to space limitation, the preassembly was performed outdoors. The influence of the temperature cannot be ignored. Using 3D coordinate value measured by API, the 3D shape analysis technique tries to simulate the erection of the steel tower and give the errors of the axis, the vertical degree and the slab staggering etc. In order to realize the goal, the computer program has been work out and verified. The satisfied results were obtained.

\section{Conclusions}

Along with the progress of social technology conditions and requirements of large scale, the steel tower segment mechanical cutting technique has been greatly developed. Aiming at the precision control of segment fabrication of the steel tower, it is very significate to comprehensive use 3D measuring technique, computer controlled hydraulic support system and precision cutting technique. On this basis, the unified computer processing precision control system should be established. The engineering practice shows that the technology can ensure the steel tower single segment precision and preassembly precision, a good condition for the realization of the overall alignment of the tower column control is provided.

\section{Acknowledgements}

This work was financially supported by Beijing Education Commission science \& technology planning project and the student science \& technology activities of North China University of Technology.

\section{References}

[1] LI You-xiang, LIU Wen, Seismic response spectrum analysis of Long-Span Bridge. Journal of Anhui Institute of Architecture \&Industry. Vol. 20 (2012), p. 18-21.

[2] YABUNO Masashi, FUJIWARA Toru, SUMI Kazuo, NOSE Takash, SUZUKI Masanao . Design of "Tatara Bridge”. IHI Engineering Review, Vol. 36 (2003), p. 40-56.

[3] SATOU Yoshiyuki, HIRAHARA Nobuyuki, MURATA Tomoji, ASANO Masatake. Erection of "Tatara Bridge". IHI Engineering Review, Vol. 36(2003), p. 65-84.

[4] WANG Hui, FANG Xing, etal. 3D Simulation of Steel Tower Segment Preassembly of Large-Scale Cable-Supported Bridge. China Railway Science, Vol. 29 (2008), p. 67-71.

[5] Japan Highway Public Corporation. Report on Fabrication of Kanmonkyo Bridge, 1977

[6] Yasuo Yakeno, etal. Development of Comprehensive Precision Control System for Long Spanned Bridge Tower Fabriction. Ishikawajiama-Harima Engineering Review. Vol. 32 (1992), p. $75-82$

[7] Hirohito kaji, et al. Report on Fabrication of Main tower for Akashi-kaikyo Bridge. Yokogawa Bridge Group Technical Report, Vol. 24, (1995), p. 82-92.

[8] UESUGI Hideo, HIRAHARA Nobuyuki, MURATA Tomoji, NOSE Takashi. Fabrication of "Tatara Bridge". IHI Engineering Review, Vol. 36 (2003), p. 57-64.

[9] Wang Hui. The application of computer aided shape simulation technique in steel tower segment fabrication of Nanjing 3rd Yangtze River Bridge. 9th International Conference on Computer-Aided Industrial Design and Conceptual Design: Multicultural Creation and Design CAIDCD, p. 665-667. (2008) 